\title{
Effects of a 14-Day Hydration Intervention on Individuals with Habitually Low Fluid Intake
}

\author{
Aaron R. Caldwell ${ }^{a}$ Megan E. Rosa-Caldwell ${ }^{a}$ Carson Keeter $^{b}$ \\ Evan C. Johnson ${ }^{b}$ François Péronnet ${ }^{c}$ Matthew S. Ganio ${ }^{a}$
}

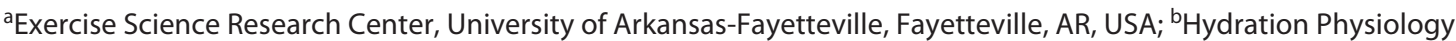
Laboratory, University of Wyoming, Laramie, WY, USA; ' $Q$ QC, Université de Montréal, Montréal, QC, Canada

\section{Keywords}

Body water · Fluid regulation · Hypohydration · Water intake

\begin{abstract}
Background: Debate continues over whether or not individuals with low total water intake (TWI) are in a chronic fluid deficit (i.e., low total body water) [1]. When women with habitually low TWI (1.6 $\pm 0.5 \mathrm{~L} /$ day) increased their fluid intake (3.5 \pm 0.1 L/day) for 4 days 24-h urine osmolality decreased, but there was no change in body weight, a proxy for total body water (TBW) [2]. In a small $(n=5)$ study of adult men, there were no observable changes in TBW, as measured by bioelectrical impedance, after increasing TWI for 4 weeks [3]. However, body weight increased and salivary osmolality decreased indicating that the study may have been underpowered to detect changes in TBW. Further, no studies to date have measured changes in blood volume (BV) when TWI is increased. Objectives: Therefore, the purpose of this study was to identify individuals with habitually low fluid intake and determine if increasing TWI, for 14 days, resulted in changes in TBW or BV. Methods: In order to identify individuals with low TWI, 889 healthy adults were screened. Participants with a self-reported TWI less than $1.8 \mathrm{~L} /$ day (men) or $1.2 \mathrm{~L} /$ day (women), and a 24-h urine osmolality greater than
\end{abstract}

karger@karger.com www.karger.com/anm

Karger $\frac{1}{\%}$
(C) 2021 The Author(s)

Published by S. Karger AG, Basel

This article is licensed under the Creative Commons AttributionNonCommercial-NoDerivatives 4.0 International License (CC BYNC-ND) (http://www.karger.com/Services/OpenAccessLicense) Usage and distribution for commercial purposes as well as any distribution of modified material requires written permission.
$800 \mathrm{mOsm}$ were included in the intervention phase of the study. For the intervention phase, 15 participants were assigned to the experimental group and 8 participants were assigned to the control group. The intervention period lasted for 14 days and consisted of 2 visits to our laboratory: one before the intervention (baseline) and 14 days into the intervention (14-day follow-up). At these visits, BV was measured using a CO-rebreathe procedure and deuterium oxide $\left(\mathrm{D}_{2} \mathrm{O}\right)$ was administered to measure TBW. Urine samples were collected immediately prior, and $3-8 \mathrm{~h}$ after the $\mathrm{D}_{2} \mathrm{O}$ dose to allow for equilibration. Prior to each visit, participants collected 24-h urine to measure 24-h hydration status. After the baseline visit, the experimental group increased their TWI to 3.7 L for males and 2.7 L for females in order to meet the current Institute of Medicine recommendations for TWI. Results: Twenty-four-hour urine osmolality decreased $(-438.7$ $\pm 362.1 \mathrm{mOsm} ; p<0.001)$ and urine volume increased $(1,526$ $\pm 869 \mathrm{~mL} ; p<0.001)$ in the experimental group from baseline, while there were no differences in osmolality $(-74.7 \pm$ $572 \mathrm{mOsm} ; p=0.45)$, or urine volume $(-32 \pm 1,376 \mathrm{~mL} ; p=$ 0.89 ) in the control group. However, there were no changes in BV (Fig. 1a) or changes in TBW (Fig. 1b) in either group. Conclusions: Increasing fluid intake in individuals with habitually low TWI increases 24-h urine volume and decreases

Note: Part of the Young Researcher Award Abstract Submission.
Correspondence to:

Aaron R. Caldwell, arcaldwell49@gmail.com 


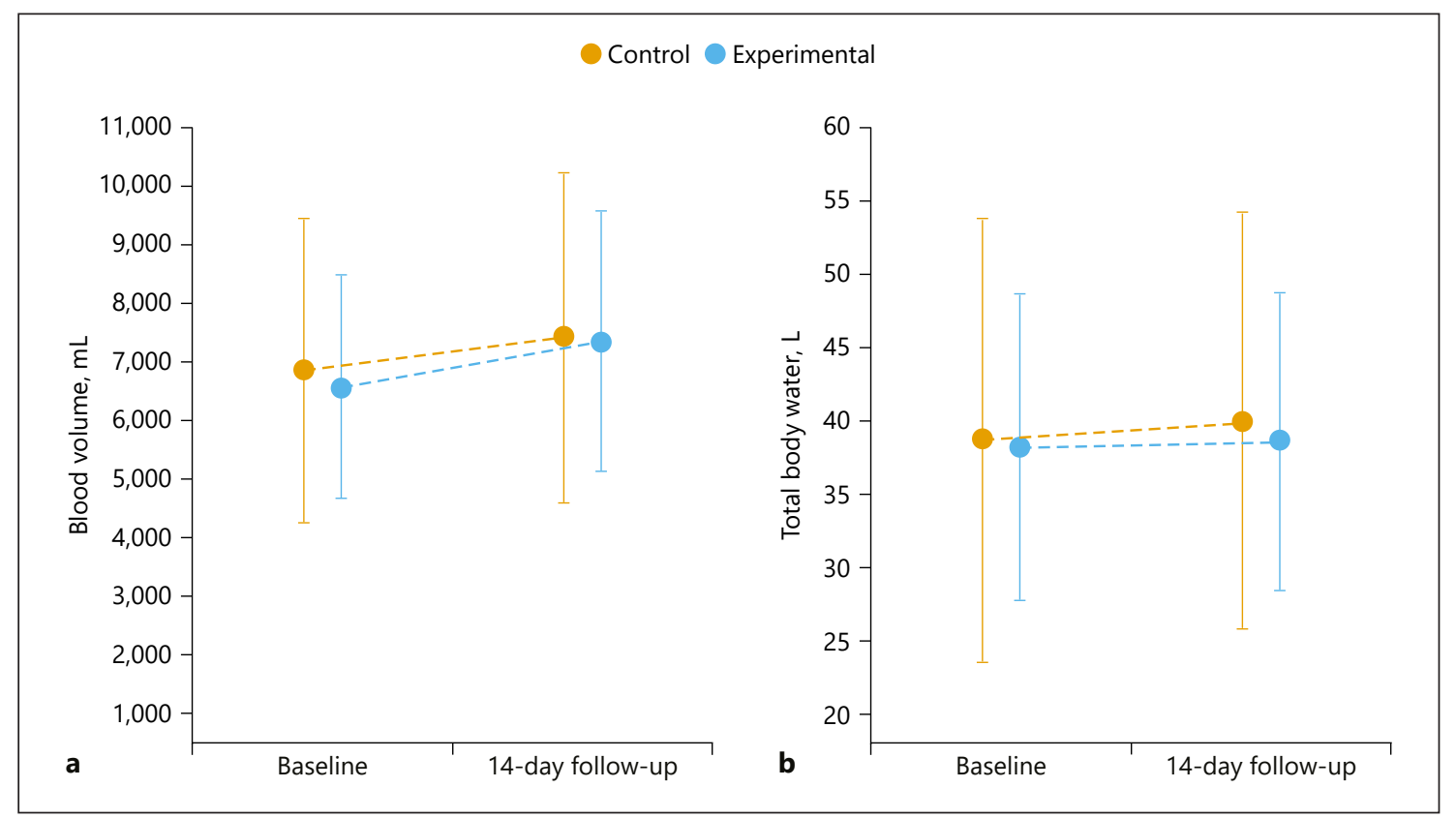

Fig. 1. BV (a) and TBW (b), over the course of the intervention. BV, blood volume; TBW, total body water.

urine osmolality but does not result in changes in TBW or BV. These findings are in agreement with previous work indicating that TWI interventions lasting 3 days [2] to 4 weeks [3] do not result in changes in TBW. Current evidence would suggest that the benefits of increasing TWI are not related changes in TBW.

(c) 2021 The Author(s)

Published by S. Karger AG, Basel

\section{Statement of Ethics}

The protocol was approved by the Institutional Review Board (IRB\#: 17-02-508) at the University of Arkansas-Fayetteville and all research participants were required to provide written informed consent for each part of the study before participation by signing a document that was approved by the University's Institutional Review Board.

\section{Conflict of Interest Statement}

E.C.J., A.R.C., and F.P. have received travel expenses and registration fees from Danone Research to attend the 2019 Hydration for Health Scientific Conference. E.C.J. serves as a consultant to Danone Research, is a member of the Hydration for Health Scientific Committee since December 2020 and previously conducted human research studies that were funded by Danone Research. M.E.R, C.K., and M.S.G. have no conflicts of interest to declare.

\section{Author Contributions}

All authors substantial contributions to the conception or design of the work (A.R.C., E.C.J., and M.S.G.); or the acquisition (A.R.C. and M.E.R.C.), analysis (A.R.C. and C.K.), or interpretation of data for the work (all authors), drafting the this abstract, and final approval of the version to be published.

\section{References}

1 Cheuvront SN, Kenefick RW. Am I drinking enough? Yes, no, and maybe. J Am Coll Nutr. 2016;35(2):185-92.

2 Johnson EC, Muñoz CX, Le Bellego L, Klein A, Casa DJ, Maresh CM, et al. Markers of the hydration process during fluid volume modification in women with habitual high or low daily fluid intakes. Eur J Appl Physiol. 2015; 115(5):1067-74.
3 Stookey JD, Hamer J, Killilea DW. Change in hydration indices associated with an increase in total water intake of more than $0.5 \mathrm{~L} /$ day, sustained over 4 weeks, in healthy young men with initial total water intake below $2 \mathrm{~L}$ /day. Physiol Rep. 2017;5(22):e13356. 